

\title{
POSSIBLE CONTRACTIONS OF QUANTUM ORTHOGONAL GROUPS
}

\author{
N.A.Gromov, I.V. Kostyakov, V.V. Kuratov \\ Department of Mathematics, \\ Syktyvkar Branch of IMM UrD RAS, \\ e-mail:gromov@dm.komisc.ru
}

October 24, 2018

\begin{abstract}
Possible contractions of quantum orthogonal groups which correspond to different choices of primitive elements of Hopf algebra are considered and all allowed contractions in Cayley-Klein scheme are obtained. Quantum deformations of kinematical groups have been investigated and have shown that quantum analog of (complex) Galilei group $G(1,3)$ do not exist in our scheme.
\end{abstract}

\section{Introduction}

Contraction of Lie groups (algebras) is a method of obtaining new Lie groups (algebras) from some initial ones with the help of passage to the limit [1]. One may define contraction of algebraic structure $(M, *)$ as the map $\phi_{\epsilon}:(M, *) \rightarrow$ $\left(N, *^{\prime}\right)$, where $(N, *)$ is algebraic structure of the same type, isomorphic to $(M, *)$ for $\epsilon \neq 0$ and nonisomorphic to the initial one for $\epsilon=0$. Except for Lie group (algebra) contractions, graded contractions [2, 3] are known, which preserve the grading of Lie algebra. Under contractions of bialgebra [4] Lie algebra structure and cocommutator are conserved. Hopf algebra (or quantum group) contractions are introduced in such a way [5, 6], that in 
the limit $\epsilon \rightarrow 0$ a new expressions for coproduct, counit and antipode are consistent with Hopf algebra axioms.

Contractions as a passage to limit are corresponded with a physical intuition. At the same time it is desirable to investigate contractions of an algebraic structures with the help of pure algebraic tools. It is possible for classical and quantum groups and algebras if one take into consideration Pimenov algebra $\mathbf{D}(\iota)$ with nilpotent commutative generators [7].

In present paper contractions of quantum orthogonal groups are studed and the groups under consideration are regarded according to [8] as an algebra of noncommutative functions but with nilpotent generators. From the contraction viewpoint Hopf algebra structure of quantum orthogonal group is more rigid as compared with the group one. Possible contractions are essentially depend on the choice of primitive elements of Hopf algebra. We have regarded all variants of such choise for quantum orthogonal group $S O_{q}(N)$ and for each variant have find all admissible contractions in Cayley-Klein scheme.

\section{Orthogonal Cayley-Klein groups}

Let us define Pimenov algebra $\mathbf{D}_{n}(\iota ; \mathbb{C})$ as an associative algebra with unit over complex number field and with nilpotent commutative generators $\iota_{k}, \iota_{k}^{2}=$ $0, \iota_{k} \iota_{m}=\iota_{m} \iota_{k} \neq 0, k \neq m, k, m=1, \ldots, n$. The general element of $\mathbf{D}_{n}(\iota ; \mathbb{C})$ is in the form

$$
d=d_{0}+\sum_{p=1}^{n} \sum_{k_{1}<\ldots<k_{p}} d_{k_{1} \ldots k_{p}} \iota_{k_{1}} \ldots \iota_{k_{p}}, \quad d_{0}, d_{k_{1} \ldots k_{p}} \in \mathbb{C} .
$$

It is possible to define the division of nilpotent generator $\iota_{k}$ by itself, namely: $\iota_{k} / \iota_{k}=1, k=1, \ldots, n$. Let us stress that the division of different nilpotent generators $\iota_{k} / \iota_{p}, k \neq p$, as well as the division of complex number by nilpotent generators $a / \iota_{k}, a \in \mathbb{C}$ are not define.

Let $S O(N ; \mathbb{C})$ be an orthogonal matrix group. Its elements are matrices $A=\left(a_{k p}\right) \in M_{N}(\mathbb{C}), A^{t}=A^{-1}$ and under the action $y^{\prime}=A y$ on vectors $y$ of complex vector space $O_{N}$ the quadratic form $y^{t} y=\sum_{k=1}^{N} y_{k}^{2}$ is preserved, where $y_{k}$ are Cartesian components of $y$. Sometimes it is convenient to regard an orthogonal group in so-called "symplectic" basis. Transformation 
from Cartesian to symplectic basis $x=D y$ is made by matrix $D$, which is a solution of equation

$$
D^{t} C_{0} D=I,
$$

where $C_{0} \in M_{N},\left(C_{0}\right)_{i k}=\delta_{i, k^{\prime}}, k^{\prime}=N+1-k$ Equation (2) has many solutions, take one of them, namely

$$
D=\frac{1}{\sqrt{2}}\left(\begin{array}{ccc}
I & 0 & -i \tilde{C}_{0} \\
0 & \sqrt{2} & 0 \\
\tilde{C}_{0} & 0 & i I
\end{array}\right), N=2 n+1
$$

where $n \times n$ matrix $\tilde{C}_{0}$ is similar to $C_{0}$. For $N=2 n$ the matrix $D$ is given by (3) but without middle column and row. Matrices $B$ of $S O(N ; \mathbb{C})$ in symplectic basis are obtained from $A$ by similarity transformation $B=D A D^{-1}$ and are subject of orthogonality relations $B^{t} C_{0} B=C_{0}$. The quadratic form $x^{t} C_{0} x$ is invariant under the action $x^{\prime}=B x$.

Complex orthogonal Cayley-Klein group $S O(N ; j ; \mathbb{C})$ is defined as the group of transformations $\xi^{\prime}(j)=A(j) \xi(j)$ of complex vector space $O_{N}(j)$ with Cartesian coordinates $\xi^{t}(j)=\left(\xi_{1},(1,2) \xi_{2}, \ldots,(1, N) \xi_{N}\right)^{t}$, which preserve the quadratic form $\operatorname{inv}(j)=\xi^{t}(j) \xi(j)=\xi_{1}^{2}+\sum_{k=2}^{N}(1, k)^{2} \xi_{k}^{2}$, where $j=\left(j_{1}, \ldots, j_{N-1}\right)$, each parameter $j_{k}$ takes two values: $j_{r}=1, \iota_{r}, r=$ $1, \ldots, N-1$, and

$$
(\mu, \nu)=\prod_{l=\min (\mu, \nu)}^{\max (\mu, \nu)-1} j_{l}, \quad(\mu, \mu)=1
$$

Let us stress, that Cartesian coordinates of $O_{N}(j)$ are special elements of Pimenov algebra $\mathbf{D}_{N-1}(j ; \mathbb{C})$. Cayley-Klein group $S O(N ; j ; \mathbb{C})$ in turn may be realised as an matrix group whose elements are taken from algebra $\mathbf{D}_{N-1}(j ; \mathbb{C})$ and consist of the $N \times N$ matrices $A(j)(A(j))_{k p}=(k, p) a_{k p}, a_{k p} \in \mathbb{C}$. Matrices $A(j)$ are subject of the additional $j$-orthogonality relations $A(j) A^{t}(j)=$ $A^{t}(j) A(j)=I$.

The passage to the symplectic description is made by matrices, which are solutions of equations (2). Let us regard the matrix $D_{\sigma}=D V_{\sigma}$, where $V_{\sigma} \in M_{N},\left(V_{\sigma}\right)_{i k}=\delta_{\sigma_{i}, k}$, and $\sigma \in S(N)$ is a permutation of the $N$-th order. It is easy to verify that $D_{\sigma}$ is again a solution of equation (2). Then in symplectic basis the orthogonal Cayley Klein group $S O(N ; j ; \mathbb{C})$ is described 
by the matrices $B_{\sigma}(j)=D_{\sigma} A(j) D_{\sigma}^{-1}$ with the additional relations of $j$ orthogonality $B_{\sigma}(j) C_{0} B_{\sigma}^{t}(j)=B_{\sigma}^{t}(j) C_{0} B_{\sigma}(j)=C_{0}$.

It should be noted that for orthogonal groups $(j=1)$ the use of different matrices $D_{\sigma}$ has no sense because of all Cartesian coordinates of $O_{N}$ are equivalent up to a choice of its enumerations. The different situation is for Cayley-Klein groups $(j \neq 1)$. Cartesian coordinates $(1, k) \xi_{k}, k=1, \ldots, N$ for nilpotent values of some or all parameters $j_{k}$ are different elements of the algebra $D_{N-1}(j ; \mathbb{C})$, therefore the same group $S O(N ; j ; \mathbb{C})$ may be realized by matrices $B_{\sigma}$ with a different disposition of nilpotent generators among their elements. Matrix elements of $B_{\sigma}(j)$ are as follows

$$
\begin{aligned}
& \left(B_{\sigma}\right)_{n+1, n+1}=b_{n+1, n+1}, \\
& \left(B_{\sigma}\right)_{k k}=b_{k k}+i \tilde{b}_{k k}\left(\sigma_{k}, \sigma_{k^{\prime}}\right), \quad\left(B_{\sigma}\right)_{k^{\prime} k^{\prime}}=b_{k k}-i \tilde{b}_{k k}\left(\sigma_{k}, \sigma_{k^{\prime}}\right), \\
& \left(B_{\sigma}\right)_{k k^{\prime}}=b_{k^{\prime} k}-i \tilde{b}_{k^{\prime} k}\left(\sigma_{k}, \sigma_{k^{\prime}}\right), \quad\left(B_{\sigma}\right)_{k^{\prime} k}=b_{k^{\prime} k}+i \tilde{b}_{k^{\prime} k}\left(\sigma_{k}, \sigma_{k^{\prime}}\right), \\
& \left(B_{\sigma}\right)_{k, n+1}=b_{k, n+1}\left(\sigma_{k}, \sigma_{n+1}\right)-i \tilde{b}_{k, n+1}\left(\sigma_{n+1}, \sigma_{k^{\prime}}\right), \\
& \left(B_{\sigma}\right)_{k^{\prime}, n+1}=b_{k, n+1}\left(\sigma_{k}, \sigma_{n+1}\right)+i \tilde{b}_{k, n+1}\left(\sigma_{n+1}, \sigma_{k^{\prime}}\right), \\
& \left(B_{\sigma}\right)_{n+1, k}=b_{n+1, k}\left(\sigma_{k}, \sigma_{n+1}\right)+i \tilde{b}_{n+1, k}\left(\sigma_{n+1}, \sigma_{k^{\prime}}\right), \\
& \left(B_{\sigma}\right)_{n+1, k^{\prime}}=b_{n+1, k}\left(\sigma_{k}, \sigma_{n+1}\right)-i \tilde{b}_{n+1, k}\left(\sigma_{n+1}, \sigma_{k^{\prime}}\right), \quad k \neq p, \\
& \left(B_{\sigma}\right)_{k p}=b_{k p}\left(\sigma_{k}, \sigma_{p}\right)+b_{k p}^{\prime}\left(\sigma_{k^{\prime}}, \sigma_{p^{\prime}}\right)+i \tilde{b}_{k p}\left(\sigma_{k}, \sigma_{p^{\prime}}\right)-i \tilde{b}_{k p}^{\prime}\left(\sigma_{k^{\prime}}, \sigma_{p}\right), \\
& \left(B_{\sigma}\right)_{k p^{\prime}}=b_{k p}\left(\sigma_{k}, \sigma_{p}\right)-b_{k p}^{\prime}\left(\sigma_{k^{\prime}}, \sigma_{p^{\prime}}\right)-i \tilde{b}_{k p}\left(\sigma_{k}, \sigma_{p^{\prime}}\right)-i \tilde{b}_{k p}^{\prime}\left(\sigma_{k^{\prime}}, \sigma_{p}\right), \\
& \left(B_{\sigma}\right)_{k^{\prime} p}=b_{k p}\left(\sigma_{k}, \sigma_{p}\right)-b_{k p}^{\prime}\left(\sigma_{k^{\prime}}, \sigma_{p^{\prime}}\right)+i \tilde{b}_{k p}\left(\sigma_{k}, \sigma_{p^{\prime}}\right)+i \tilde{b}_{k p}^{\prime}\left(\sigma_{k^{\prime}}, \sigma_{p}\right), \\
& \left(B_{\sigma}\right)_{k^{\prime} p^{\prime}}=b_{k p}\left(\sigma_{k}, \sigma_{p}\right)+b_{k p}^{\prime}\left(\sigma_{k^{\prime}}, \sigma_{p^{\prime}}\right)-i \tilde{b}_{k p}\left(\sigma_{k}, \sigma_{p^{\prime}}\right)+i \tilde{b}_{k p}^{\prime}\left(\sigma_{k^{\prime}}, \sigma_{p}\right) .
\end{aligned}
$$

Here $b, b^{\prime}, \tilde{b}, \tilde{b}^{\prime} \in \mathbb{C}$ may be easily expressed by matrix elements of $A$.

\section{Contractions of quantum orthogonal groups.}

\subsection{Formal definition of the quantum group $S O_{v}(N ; j ; \sigma)$}

The starting point of the definition of quantum groups 8 is an algebra $\mathbb{C}\left\langle T_{i k}\right\rangle$ of noncommutative polynomials of $N^{2}$ variables. We start with an algebra $\mathbf{D}\left\langle\left(T_{\sigma}\right)_{i k}\right\rangle$ of noncommutative polynomials of $N^{2}$ variables, which are an elements of the direct product $\mathbf{D}_{N-1}(j) \otimes \mathbb{C}\left\langle t_{i k}\right\rangle$. More precisely the elements $\left(T_{\sigma}\right)_{i k}$ are obtained from the elements $\left(B_{\sigma}(j)\right)_{i k}$ of equations (5) by the replacement of commutative variables $b, b^{\prime}, \tilde{b}, \tilde{b}^{\prime}$ with the noncommutative variables $t, t^{\prime}, \tau, \tau^{\prime}$, respectively. One introduce additionally the transformation of the deformation parameters $q=e^{z}$ as follows: $z=J v$, where $v$ is 
a new deformation parameter and $J$ is some product of parameters $j$ for the present unknown. Let $\tilde{R}_{v}(j), C(j)$ are matrices which are obtained from corresponding matrices of [8] by the replecement of deformation parameter $z$ with $J v$ :

$$
R_{v}(j)=R_{q}(z \rightarrow J v), \quad C(j)=C(z \rightarrow J v) .
$$

The commutation relations of the generators $T_{\sigma}(j)$ are defined by

$$
R_{v}(j) T_{1}(j) T_{2}(j)=T_{2}(j) T_{1}(j) R_{v}(j),
$$

where $T_{1}(j)=T_{\sigma}(j) \otimes I, T_{2}(j)=I \otimes T_{\sigma}(j)$ and the additional relations of $(v, j)$-orthogonality

$$
T_{\sigma}(j) C(j) T_{\sigma}^{t}(j)=T_{\sigma}^{t}(j) C(j) T_{\sigma}(j)=C(j)
$$

are imposed.

One define the quantum orthogonal Cayley-Klein group $S O_{v}(N ; j ; \sigma)$ as the quotient algebra of $\mathbf{D}\left\langle\left(T_{\sigma}\right)_{i k}\right\rangle$ by relations (7), (8). Formally $S O_{v}(N ; j ; \sigma)$ is a Hopf algebra with the following coproduct $\Delta$, counit $\epsilon$ and antipode $S$ :

$$
\Delta T_{\sigma}(j)=T_{\sigma}(j) \dot{\otimes} T_{\sigma}(j), \quad \epsilon\left(T_{\sigma}(j)\right)=I, \quad S\left(T_{\sigma}(j)\right)=C(j) T_{\sigma}^{t}(j) C^{-1}(j) .
$$

As far as only second diagonal elements of the matrix $\mathrm{C}$ are different from zero and for $q=1$ this matrix is equal to $C_{0}$, then we have the symplectic description of $S O_{q}(N)$.

\subsection{Allowed contractions of $S O_{v}(N ; j ; \sigma)$}

The formal definition of $S O_{v}(N ; j ; \sigma)$ should be a real definition of quantum group, if the proposed construction is a consistent Hopf algebra structure under nilpotent values of some or all parameters $j$. Counit $\epsilon\left(t_{n+1, n+1}\right)=$ $1, \epsilon\left(t_{k k}\right)=1, k=1, \ldots, n$ and $\epsilon(t)=\epsilon(\tau)=0$ for the rest generators do not restrict the values of $j$. Parameters $j$ are arranged in the expressions for coproduct $\Delta$ exactly as in matrix product of $B_{\sigma}(j)$, and as far as the last ones form the group $S O(N ; j ; \mathbb{C})$ for any values of $j$, then no restrictions follow from the coproduct. Different situation is with the antipode $S$. Really, for

elements $\left(T_{\sigma}\right)_{k^{\prime} k}=t_{k^{\prime} k}+i \tau_{k^{\prime} k}\left(\sigma_{k}, \sigma_{k^{\prime}}\right), \quad k=1, \ldots, n$, antipode is obtained as

$$
S\left(\left(T_{\sigma}\right)_{k^{\prime} k}\right)=\left(T_{\sigma}\right)_{k^{\prime} k} \cdot e^{2 J \rho_{k} v}
$$


and depend both on $\rho_{k}$ and for the present undetermined factor $J$. Antipode is an antihomomorphism of Hopf algebra and therefore have to transform $T_{\sigma}(j)$ to a matrix with the same distribution of the nilpotent parameters $j$ in its elements, i.e. the right and the left parts of equation (10) must be identical elements of $\mathbf{D}_{N-1}(j) \otimes \mathbb{C}\left\langle t_{i k}\right\rangle$. For $J=1$ this condition is holds for any values of the parameters $j$. The case $J \neq 1$ require additional discussion.

Next condition which must be taken into account is the $(v, j)$-orthogonality relations (8). We require that the number of equations in (8) is not changed as compared with the initial quantum group. It is possible when nilpotent generators are appeared in equation (8) either with the powers greater or equal two (and then the corresponding terms are equal to zero) or as homogeneous multipliers. Taking into account all these arguments and using the explicit expressions for antipode and $(v, j)$-orthogonality we can find possible contractions of quantum orthogonal groups, which are described by the following theorems.

Theorem 1. If the deformation parameter is not transformed $J=$ 1 , then the following maximal $n$-dimensional contraction of the orthogonal quantum group $S O_{v}(N ; j ; \sigma), \quad N=2 n+1$ is allowed: $j_{2 s}=\iota_{2 s}, \quad s=$ $1, \ldots, m, j_{2 r+1}=\iota_{2 r+1}, r=m, \ldots, n-1,0 \leq m \leq n$, for example, for permutation $\sigma: \sigma_{n+1}=2 m+1, \sigma_{s}=2 s-1, \sigma_{s^{\prime}}=2 s, s=1, \ldots, m, \sigma_{r}=$ $2 r, \sigma_{r^{\prime}}=2 r+1, r=m+1, \ldots, n$.

Theorem 2. If the deformation parameter is not transformed $J=1$, then the following maximal n-dimensional contraction of the quantum orthogonal group $S O_{v}(N ; j ; \sigma), N=2 n$ is allowed: $j_{2 s}=\iota_{2 s}, s=1, \ldots, m-$ $1, j_{2 p-1}=\iota_{2 p-1}, p=m, \ldots, u, j_{2 r}=\iota_{2 r}, r=u, \ldots, n-1,1 \leq m \leq u \leq n$, for example, for permutation $\sigma: \sigma_{n}=2 m-1, \sigma_{n^{\prime}}=2 u, \sigma_{s}=2 s-1, \sigma_{s^{\prime}}=$ $2 s, s=1, \ldots, m-1, \sigma_{p}=2 p, \sigma_{p^{\prime}}=2 p+1, p=m, \ldots, u-1, \sigma_{r}=$ $2 r+1, \sigma_{r^{\prime}}=2 r, r=u, \ldots, n-1$.

Remark 1. It should be noted that as $\sigma$ may be taken any permutation with the properties $\left(\sigma_{k}, \sigma_{k^{\prime}}\right)=1, k=1, \ldots, n$ (or $\left.n-1\right)$.

Remark 2. Admissible contractions for number of parameters $j_{k}$ less then $n$ are obtained from theorems 1 and 2 by setting part of $j_{2 s}, j_{2 p-1}, j_{2 r}$, $j_{2 r+1}$ equal to one.

We return to the antipode (10) for $J \neq 1$. As far as $\rho_{n+1}=0$ for $N=$ $2 n+1$, and $\rho_{n}=\rho_{n^{\prime}}=0$ for $N=2 n$, we shall regard these two cases separately.

Theorem 3. If the deformation parameter is transformed $(J \neq 1)$, then 
the following contractions of the quantum orthogonal group $S O_{v}(N ; j ; \sigma)$, $N=2 n+1$ are allowed:

1. For $J=j_{n+1}$, a) $j_{n+1}=\iota_{n+1}$, if $1<\sigma_{n+1}<n+1$; b) $j_{n+1}=$ $\iota_{n+1}, j_{1}=1, \iota_{1}$, if $\sigma_{n+1}=1$.

2. For $J=j_{n}$, a) $j_{n}=\iota_{n}$, if $n+1<\sigma_{n+1}<2 n+1$; b) $j_{n}=\iota_{n}, j_{2 n}=$ $1, \iota_{2 n}$, if $\sigma_{n+1}=2 n+1$.

3. For $J=j_{n} j_{n+1}, j_{n}=1, \iota_{n}, j_{n+1}=1, \iota_{n+1}$, if $\sigma_{n+1}=n+1$.

Theorem 4. If the deformation parameter is transformed $(J \neq 1)$, then the following contractions of the quantum orthogonal group $S O_{v}(N ; j ; \sigma)$, $N=2 n$ are allowed:

1. For $J=j_{n}$, a) $j_{n}=\iota_{n}$, if $\sigma_{n}>1, \sigma_{n^{\prime}}<2 n$; b) $j_{n}=\iota_{n}, j_{1}=1, \iota_{1}$, if $\sigma_{n}=1, \sigma_{n^{\prime}}<2 n$; c) $j_{n}=\iota_{n}, j_{2 n-1}=1, \iota_{2 n-1}$, if $\sigma_{n}>1, \sigma_{n^{\prime}}=2 n$; d) $j_{n}=\iota_{n}, j_{1}=1, \iota_{1}, j_{2 n-1}=1, \iota_{2 n-1}$, if $\sigma_{n}=1, \sigma_{n^{\prime}}=2 n$.

2. For $J=j_{n-1}$. a) $j_{n-1}=\iota_{n-1}$, if $\sigma_{n^{\prime}}<2 n$; b) $j_{n-1}=\iota_{n-1}, j_{2 n-1}=$ $1, \iota_{2 n-1}$, if $\sigma_{n}<2 n-1, \sigma_{n^{\prime}}=2 n$; c) $j_{n-1}=\iota_{n-1}, j_{2 n-2}=1, \iota_{2 n-2}, j_{2 n-1}=$ $1, \iota_{2 n-1}$, if $\sigma_{n}=2 n-1, \sigma_{n^{\prime}}=2 n$.

3. For $J=j_{n+1}$. a) $j_{n+1}=\iota_{n+1}$, if $\sigma_{n}>1$; b) $j_{n+1}=\iota_{n+1}, j_{1}=1, \iota_{1}$, if $\sigma_{n}=1, \sigma_{n^{\prime}}>2$; c) $j_{n+1}=\iota_{n+1}, j_{1}=1, \iota_{1}, j_{2}=1, \iota_{2}$, if $\sigma_{n}=1, \sigma_{n^{\prime}}=2$.

4. For $J=j_{n-1} j_{n}$. a) $j_{n-1}=\iota_{n-1}, j_{n}=\iota_{n}$, if $\sigma_{n^{\prime}}<2 n$;b) $j_{n-1}=$ $\iota_{n-1}, j_{n}=\iota_{n}, j_{2 n-1}=1, \iota_{2 n-1}$, if $\sigma_{n^{\prime}}=2 n$.

5. For $J=j_{n} j_{n+1}$. a) $j_{n}=\iota_{n}, j_{n+1}=\iota_{n+1}$, if $\sigma_{n}>1$; b) $j_{1}=1, \iota_{1}, j_{n}=$ $\iota_{n}, j_{n+1}=\iota_{n+1}$, if $\sigma_{n}=1$.

6. For $J=j_{n-1} j_{n} j_{n+1}$. a) $j_{n-1}=1, \iota_{n-1}, j_{n}=1, \iota_{n}, j_{n+1}=1, \iota_{n+1}$, if $\sigma_{n}=n, \sigma_{n^{\prime}}=n+1$.

Hopf algebra $S O_{q}(N ; j ; \sigma), N=2 n+1$ has $n$ primitive elements which correspond to $n$ diagonal $2 \times 2$ submatricies: $\operatorname{diag}\left(\left(B_{\sigma}\right)_{k k},\left(B_{\sigma}\right)_{k^{\prime} k^{\prime}}\right)=\operatorname{diag}\left(b_{k k}+\right.$ $\left.i \tilde{b}_{k k}\left(\sigma_{k}, \sigma_{k^{\prime}}\right), b_{k k}-i \tilde{b}_{k k}\left(\sigma_{k}, \sigma_{k^{\prime}}\right)\right), k=1, \ldots, n$, see (5). If the deformation parameter $z$ is fixed $(J=1)$ under contractions, then all primitive elements of the contracted quantum orthogonal group are corresponded to Euclidean rotation $S O(2)$. If the deformation parameter is transformed $z=$ $\iota v$, then all primitive elements are correspond to Galilei transformation $S O(2 ; j=\iota)=G(1,1)$. The same is true for the contracted quantum groups $S O_{q}(N ; j ; \sigma), N=2 n$. Let us note that contractions of quantum orthogonal algebras with a different sets of primitive elements have been discussed in [四], 9].

Quantum orthogonal groups have contractions with the same nilpotent parameters $j$ both with fixed deformation parameter and with transformed 
one. For example, quantum group $S O_{q}(2 n+1 ; j ; \sigma)$ for even $n=2 p$ at $\sigma_{n+1}=$ 1 according with theorem 1 has contraction $j_{n}=\iota_{n}, j_{n+1}=\iota_{n+1}, J=1$ and according with item 3 of theorem 3 has the same two dimensional contraction, but $J=\iota_{n} \iota_{n+1}$. Let us stress that the cases $J=1$ and $J \sim \iota$ are realized for different sets of primitive elements in Hopf algebra.

Let permutation $\sigma$ is identical, i.e. $\sigma_{k}=k, \sigma_{k^{\prime}}=k^{\prime}, \sigma_{n+1}=n+1$. It follows from theorems 1 and 2 that there are no contractions of $S O_{q}(N ; j)$ with fixed deformation parameter $(J=1)$. For $N=2 n+1$ from theorem 3 we obtain three possible contractions: $j_{n}=1, \iota_{n}, j_{n+1}=1, \iota_{n+1}$ (both parameters $j_{n}$ and $j_{n+1}$ independently take nilpotent values) and deformation parameters is transformed with $J=j_{n} j_{n+1}$. For $N=2 n$ from theorem 4 we obtain seven admissible contractions: $j_{n-1}=1, \iota_{n-1}, j_{n}=1, \iota_{n}, j_{n+1}=1, \iota_{n+1}$, where deformation parameter is multiplied by $J=j_{n-1} j_{n} j_{n+1}$. It should be considered in [10] just these allowed contractions.

From the contraction viewpoint Hopf algebra structure of quantum orthogonal group is more rigid as compared with a group one. Cayley-Klein groups are obtained from $S O(N ; j)$ for all nilpotent values of parameters

$j_{k}, k=1, \ldots, N-1$, whereas their quantum deformations exist only for some of them $\left(\leq\left[\frac{N}{2}\right]\right)$. It should be note that among the contracted for equal number of parameters $j$ quantum orthogonal groups may be isomorphic as Hopf algebra quantum groups. Quantum group isomorphism is not regarded in this paper.

\section{Quantum complex kinematic groups}

Kinematic groups are motion groups of the maximal homogeneous four dimensional (one temporal and three space coordinates) space-time models [11]. All these groups may be obtained from the real group $S O(5 ; \mathbb{R})$ by contractions and analytic continuations [7]. There are three types of kinematics: nonrelativistic - Galilei $G(1,3)=S O\left(5 ; \iota_{1}, \iota_{2}, 1,1\right)$ with zero curvature and Newton $N^{ \pm}(1,3)=S O\left(5 ; j_{1}=1, i ; \iota_{2}, 1,1\right)$ with positive and negative curvature, respectively; relativistic — Poincare $P(1,3)=S O\left(5 ; \iota_{1}, i, 1,1\right)$ with zero curvature and (anti) de Sitter $S^{ \pm}(1,3)=S O\left(5 ; j_{1}=1, i ; i, 1,1\right)$ with (positive) negative curvature; exotic - Carroll $C^{0}(1,3)=S O\left(5 ; \iota_{1}, 1,1, \iota_{4}\right)$ with zero curvature and $C^{ \pm}(1,3)=S O\left(5 ; j_{1}=1, i ; 1,1, \iota_{4}\right)$ with positive and negative curvature. 
The groups $N^{ \pm}(1,3)$ are the real forms of the complex Newton group $N(4)$, Poincare group $P(1,3)$ is the real form of the complex Euclid group $E(4)$, the groups $C^{ \pm}(1,3)$ are the real forms of the complex Carroll group $C(4)$. In this paper the quantum deformations of the complex orthogonal groups are regarded, therefore whith the help of contractions a quantum analogs of the complex kinematic groups may be obtained. Possible contractions of the complex quantum groups $S_{q}(5 ; j ; \sigma)$ are described by theorems 1 and 3 for $N=5$. If deformation parameter remain unchanged $(J=1)$, then we have the quantum analogs of Euclead group $E_{q}(4)$, Newton group $N_{q}(4)$ and Carroll group $C_{q}(4)$. If the deformation parameter is transformed under contraction $z=\iota_{2} v$, then we have one more quantum deformation of Newton group $N_{v}(4)$, which is not isomorphic to the previous one. Two primitive elements of $N_{q}(4)$ correspond to the elliptic translation along the temporal axis $t$ and to the rotation in the space plane $\left\{r_{2}, r_{3}\right\}$ (both are isomorphic to $S O(2)$ ), while primitive elements of $N_{v}(4)$ correspond to the flat translation along the spatial axis $r_{2}$ and to Galilei boost in the space-time plane $\left\{t, r_{1}\right\}$ (both are isomorphic to Galilei group $S O\left(2 ; j_{2}=\iota_{2}\right)=G(1,1)$ ). We did not obtain the quantum deformations of the complex Galilei $G(4)$ and Carroll $C^{0}(4)$ groups.

According with correspondence principle a new physical theory must include an old one as a particle case. For space-time theory this principle is realised as the chain of limit transitions: general relativity pass to special relativity when space-time curvature tends to zero and special relativity pass to classical physics when light velosity tends to infinite. For kinematical groups this correspond to the chain of contractions:

$$
S^{ \pm}(1,3) \stackrel{K \rightarrow 0}{\longrightarrow} P(1,3) \stackrel{c \rightarrow \infty}{\longrightarrow} G(1,3) .
$$

As it was mentioned above there is no quantum deformation of the complex Galilei group, therefore it is not possible to construct the quantum analog of the full chain of contractions (11) even at the level of complex groups. This means that (at least standart) quantum deformation of the flat nonrelativistic space-time do not exist.

\section{References}

[1] E.Inönü, E.P.Wigner, Proc.Nat.Acad.Sci. USA 39, 510 (1953). 
[2] M. de Montigny, J.Patera, J.Phys.A: Math.Gen. 24, 525 (1991).

[3] R.V.Moody, J.Patera, J.Phys.A: Math.Gen. 24, 2227 (1991).

[4] A.Ballesteros, N.A.Gromov, F.J.Herranz, M.A. del Olmo, M.Santander, J.Math.Phys. 36, 5916 (1995); hep-th/9412083.

[5] E.Celeghini, R.Giachetti, E.Sorace, M.Tarlini, J.Math.Phys. 32, 1155 (1991).

[6] E.Celeghini, R.Giachetti, E.Sorace, M.Tarlini, J.Math.Phys. 32, 1159 (1991).

[7] N.A.Gromov, Contractions and Analytical Continuation of Classical Groups. Unified Approach, (Komi SC, Syktyvkar, 1990) (in Russian).

[8] N.Yu.Reshetikhin, L.A.Takhtajan, L.D.Faddeev, Algebra i Analis 1, 178 (1989).

[9] N.A.Gromov, Turkish J. Phys. 21, 3771997.

[10] N.A.Gromov, I.V.Kostyakov, V.V.Kuratov, In: Algebra, Differential Equations and Probability Theory (Komi SC, Syktyvkar, 1997), 3-29, (Trudy Komi NC, 151).

[11] H.Bacry, J.-M.Levy-Leblond, J.Math.Phys. 9, 1605 (1968). 